\title{
EL FORTALECIMIENTO DE LOS PARLAMENTOS EN LA UNIÓN EUROPEA: LA INCORPORACIÓN DEL EARLY WARNING SYSTEM
}

\author{
ELVIRO ARANDA ÁLVAREZ \\ Profesor Titular de Derecho Constitucional \\ Universidad Carlos III de Madrid \\ Diputado
}

SUMARIO

Introducción

I. Los precedentes a los Protocolos del Tratado Constitucional.

II. Informe final del Grupo IV sobre el cometido de los parlamentos nacionales en la Unión Europea.

III. Informe final del Grupo I sobre la aplicación de los principios de subsidiariedad y proporcionalidad.

IV. La opción de los Protocolos del Tratado Constitucional. La normalización de procedimiento de «alerta rápida».

V. Conclusiones.

\section{INTRODUCCIÓN}

En estos últimos tiempos en los que nos encontramos inmersos en el proceso de elaboración, aprobación y ratificación de un Tratado Constitucional para la Unión Europea se está hablando mucho de la arquitectura institucional y, en especial, del papel que juega en ella el Parlamento Europeo y los parlamentos nacionales. Es lógico que así sea, no debemos pasar por alto que está en juego no sólo un «trenzado» útil y eficiente de instituciones en una organización compleja, sino cómo se hace efectivo el principio democrático en la realidad comunitaria. Creo que no hace falta señalar que este principio está en los Tratados (art. 6.1 del TUE y art. I.2 del Tratado Constitucional), y lo que es mas importan- 
seguridad común, política de defensa, etc). En definitiva, el reconocimiento de la idea de ciudadanía europea y el importante aumento de poder de la Unión en detrimento de los Estados refuerza las tesis de aquellos que veían en la construcción europea una escasa participación de los ciudadanos y los órganos parlamentarios en cuanto depositarios de la legitimidad democrática.

Sin duda, el Tratado de la Unión será la espoleta que active el gran debate sobre la necesidad de reforzar el principio democrático en el funcionamiento de las instituciones europeas. Como decía, la transferencia de competencias de una fuerte tradición estatal (política exterior y seguridad común, cooperación estrecha en el ámbito de la Justicia y los asuntos de Interior o la Unión monetaria), junto con el compromiso del TUE por la reforma de las instituciones comunitarias para un fortalecimiento democrático, mejoran el poder del Parlamento, tanto del Europeo como la intervención de los parlamentos nacionales en las políticas de la Unión. Consolidación que se ve reforzada con el Tratado de Ámsterdam. Por ello, no faltaron quienes apuntaron que el verdadero vencedor en dicho Tratado fue el Parlamento Europeo ${ }^{4}$. Por un lado, su poder legislativo fue ampliado cuantitativa y cualitativamente. La Codecisión, introducida ya en Maastricht, se amplio a materias de la importancia de la política social y la sanidad pública. Por otro, materias que se veían en el procedimiento de Cooperación pasan al procedimiento de Codecisión: libre circulación de trabajadores, libre establecimiento, investigación, transportes, etc. Finalmente, no se debe olvidar que este Tratado también retocó el equilibrio de poderes a favor del Parlamento (el Comité de Conciliación se debe centrar en las enmiendas propuestas por el Parlamento; o que dicho Parlamento no estará obligado a informar al Consejo cuando pretenda rechazar su posición común $)^{5}$. No menos importante, son las competencias de control político que adquiere el Parlamento sobre la Comisión al tener que aprobar el nombramiento del Presidente y de los miembros de la Comisión. Consagrándose la doble investidura del Presidente de la Comisión y de la propia Comisión ante las dos fuentes de legitimidad comunitaria: los Estados miembros y el Parlamento ${ }^{6}$

La conclusión no puede ser otra más que frenar las apreciaciones «precipitadas», hechas en muchos casos desde parámetros y modelos institucionales alejados de la realidad comunitaria. Hay que tener en cuenta que después de casi cincuenta años de construcción del proyecto europeo, en la práctica, hace escasos diez años que nos estamos planteando reforzar los parlamentos como centro de legitimación democrática de la Unión Europea ${ }^{7}$. Diez años que han dado para construir la Codecisión en favor del Parlamento a la hora de producir el Derecho por parte de Europa o, entre otras, reforzar el control político que el Parlamento Europeo ejerce sobre la Comisión. También lo han hecho para el reconoci-

1 Por todos, vid. Pérez Tremps, Pablo. La débil «Parlamentarización» de la integración en España. En la obra de AA.VV. La Encrucijada constitucional de la Unión Europea. Director Eduardo García de Enterria. Civitas 2002. Págs. 401-402. y MAngas Martín, Araceli. «La reforma institucional en el Tratado de Ámsterdam». En la Revista de Derecho Comunitario Europeo. Núm. 3, 1998 enero/Junio. Págs 19 y ss.

2 Vid. Fernández Estaban, María Luisa. «La Constitución Europea y la Carta de Derechos Fundamentales». Estudios de Progreso, núm. 12 Edit. Fundación Alternativas, pág. 9.

3 La profesora Abellán Honrubia apunta en su manual de Derecho Comunitario cómo el origen de ese déficit de funcionamiento democrático se encuentra en los propios Tratados constitutivos de las Comunidades Europeas, tanto en la atribución de competencias efectuadas por los Estados miembros a la Comunidad, como en la distribución del poder entre las instituciones comunitarias. ABELlán HonRubia, Victoria y Otros. Lecciones de Derecho Comunitario Europeo. $3^{\text {a }}$ edición, revisada y actualizada. Editorial Ariel, Barcelona 1998, 
te, está en la cultura política y jurídica de todos los Estados miembros y, por supuesto, en el sentir que inspiró la aprobación del Tratado de Roma en 1957.

Por lo tanto, inicio este artículo sumándome como tantos otros han hecho al sentir común por la insatisfactoria situación en la que se encuentra la institución parlamentaria en la Unión Europea ${ }^{1}$.

Sin embargo, creo que no basta con unirse a la que es una opinión común, hasta el punto que la propia Unión Europea ha expresado en innumerables ocasiones esa mala conciencia (v.gr. Protocolo sobre el cometido de los parlamentos nacionales en la UE anexo al Tratado de Ámsterdam o la Declaración sobre el futuro de la Unión anexo al Tratado de Niza); resulta necesario analizar el problema desde la comprensión de los criterios que han regido estos años la articulación orgánica de la Unión y, por supuesto, desde la realidad histórica en la que se han construido dichas instituciones.

Lo primero que hay que recordar es que hasta llegar a la actual Unión Europea se ha tenido que recorrer un largo camino. Aunque hoy se puede hablar de supranacionalidad y se empieza a visualizar una organización política que transciende los Estados que la conforman, está claro que las Comunidad Europea se ha construido desde las relaciones internacionales de los Estados europeos. Es decir, la Comunidad es una organización internacional que crean los Estados y donde éstos tienen un protagonismo fundamental. Por ello, no es de extrañar que se pueda hablar de la coexistencia de dos tendencias en la construcción Europea: la intergubernamental, que se fija en una construcción sobre elementos típicos del Derecho internacional y la supranacional, que incide en los elementos que conforman una organización con personalidad política propia y distinta a la de los Estados que la conforman ${ }^{2}$. Precisamente, la coexistencia de estas dos tendencias es la que sostiene el equilibrio institucional de la Unión: el Consejo, que representa a los Estados; la Comisión, que hace lo propio con los intereses Comunitarios y el Parlamentos Europeo, que es el encargado de hacer presente a los ciudadanos.

Esta situación ha dado lugar a que en un primer momento, quizás hasta la firma del Acta Única, los parlamentos nacionales tenían un escaso peso en las decisiones que tomaban los órganos Comunitarios, en parte por culpa de ellos mismos que habían hecho dejación de su competencias cuando se aceptan los tratados constitutivos o modificativos ${ }^{3}$.

Con la elección por un procedimiento de sufragio universal directo del Parlamento Europeo (1978) se empieza a caer en la cuenta del déficit democrático que conlleva el sistema de funcionamiento de las instituciones de las Comunidades. Sentimiento que se agudiza con la aprobación del Acta Única en 1986 y, sobre todo, con la aprobación del Tratado de Maastricht en 1992, donde, a demás de reconocer la ciudadanía europea, se toman decisiones que afectan de lleno a poderes de los Estados (v.gr. política monetaria, política de

pág. 45. Sobre esta cuestión también, Molina Del Pozo, Carlos Francisco. Manual de Derecho de la Comunidad Europea. Edit. DIJUSA. Madrid 2002. y AldeCOA LuZArRAgA, Francisco. La Integración Europea. Edit. Tecnos. Madrid 2002.

4 Elmar Brok. European Policy Centre (ed), Making of the Ámsterdam Treaty, (1997), pág. 45.

5 Esta idea puede verse más desarrollada en Renaud. Dehousse.»La arquitectura institucional europea después de Ámsterdam: ¿sistema parlamentario o estructura regulatoria?. En la obra de AA.VV. Problemas de legitimación en la Europa de la Unión. Las respuestas del Tratado de Ámsterdam. Edición a cargo de Tomás de la Quadra-Salcedo y Antonio Estella de Noriega. Edit. Carlos III de Madrid y Boletín Oficial del Estado. Madrid 2000. Pág. 102 y 103. 
zar esa cooperación: la Conferencia de los parlamentos (o «Assises») que será consultada sobre las grandes orientaciones de la Unión Europea. Además, de que tanto el Presidente del Consejo Europeo como el Presidente de la Comisión tengan que presentar en cada sesión de la Conferencia de los parlamentos un informe sobre el estado de la Unión.

La Conferencia invita al Parlamento Europeo y a los Parlamentos nacionales a reunirse siempre que sea necesario bajo la forma de Conferencia de los Parlamentos (o «Assises》).

Se consultará a la Conferencia de los Parlamentos sobre las grandes orientaciones de la Unión Europea, sin perjuicio de las competencias del Parlamento Europeo y los derechos de los Parlamentos nacionales. El presidente del Consejo Europeo y el presidente de la Comisión presentarán en cada sesión de la Conferencia de los Parlamentos un informe sobre el estado de la Unión.

La Conferencia de los Parlamentos, aunque se enmarca en una declaración genérica para reforzar la institución parlamentaria dando protagonismo a los parlamentos nacionales y al Parlamento Europeo, es la opción de cooperación más decidida que resulta del Tratado de Maastricht, aunque como recuerda toda la doctrina no dejó de ser una forma de dar salida a una iniciativa política que parte de los Presidentes de los Parlamentos de los Estados miembros de la Comunidad y el Parlamento europeo (septiembre de 1990) convocando una «Conferencia de los Parlamentos de la Comunidad Europea» para tratar los temas que serían objeto de la Conferencia que llevaría a la Unión Política, Económica y Monetaria. La Conferencia expresó graves problemas en su conformación, funcionamiento y toma de acuerdos que hizo que tuviera escaso éxito.

Estamos ante dos declaraciones de reforzamiento del Parlamento, dos fórmulas que aunque no contradictorias sí se mostraron, en la práctica, redundantes, lo que llevó a la Conferencia de Parlamentos (que por cierto, algunos vieron como el embrión de una posible segunda cámara para Europa. Delegaciones de Bélgica y Francia) ${ }^{9}$ desaparezca del entramado institucional.

La Declaración $\mathrm{n}^{\circ} .13$ si que resultó útil para fortalecer la Conferencia de órganos especializados en asuntos europeos (COSAC), que estaba en funcionamiento desde la Conferencia de Presidentes de los Parlamentos de los Estados miembros de la Comunidad celebrada en París en 1989. Conferencia que aunque con pretensiones mucho más modestas que la «Assises» tuvo mejor fortuna, puesto que, no sólo consiguió

6 La importancia de este procedimiento de «investidura» lo hemos apreciado en los días en los que me encontraba elaborando este trabajo con las dificultades que el Presidente de la Comisión José Manuel Durào Barroso tuvo para confirmar su equipo de ante el Parlamento. Como es de todos conocidos el Parlamento se resistió a investir a dicha Comisión por la presencia en ella como Comisario de Justicia al italiano Rocco Buttiglione que había realizados ciertas declaraciones que se consideraban discriminatorias de los homosexuales y las madres solteras. El resultado final y a un día de la firma por los Jefes de Estado y Gobierno del Tratado Constitucional fue que Durào Barroso decidió retirar su propuesta de Comisión para que no se produjese la crisis que una votación en contra auguraba.

7 Sobre el fortalecimiento del Parlamento en las últimas décadas vid. CANCELA OUTDA, Celso. El proceso de constitucionalización de la Unión Europea. De Roma a Niza. Edit. Catedra Jean Monnet. Universidad de Santiago de Compostela, 2001, págs. 394 y ss.

8 Delgado-Iribarren ha señalado con acierto que Maastricht pone en el primer plano de discusión la transferencia de competencias estatales esenciales, tradicionalmente ligadas a las cualidades de la potestad soberana del Estado moderno y la presión ejercida por la opinión pública sobre algunos gobiernos lleva a que éstos, a su vez, plantearan la necesidad de establecer diferentes canales de reforzamiento del principio democrático en la organización y funcionamiento del sistema comunitario. DELGADO-IRIBARREN García-Campero, Manuel. La función de los parlamentos nacionales en la arquitectura europea. En la obra de AA.VV La encrucijada constitucional de la Unión Europea. Opus cit. pág. 373. 
miento institucional de la Conferencia de órganos especializados en asuntos europeos (COSAC) y la aprobación de varios Protocolos sobre los cometidos de los parlamentos nacionales en la Unión Europea. Entre ellos, los dos que incorpora el Tratado Constitucional; el de los cometidos de los parlamentos nacionales en la Unión Europea, que como veremos sigue la línea abierta en Maastricht de reforzar el control político de la acción de los gobiernos en el desarrollo comunitario; y el de la aplicación de los principios de subsidiariedad y proporcionalidad, que consolida un procedimiento político para que los Estados miembros y los entes territoriales subestatales puedan controlar el régimen de competencias concurrentes entre los diferentes niveles de poder.

\section{LOS PRECEDENTES A LOS PROTOCOLOS DEL TRATADO CONSTITUCIONAL}

Como decía en el apartado anterior, será en Maastricht cuando aparezca con cierto empeño la necesidad de reforzar la acción parlamentaria ${ }^{8}$. La Conferencia preparatoria del TUE estaba de acuerdo en reforzarla, sin embargo, en estos primeros momentos no se tenía muy claro como se debía articular esa participación. La asunción por parte de las Comunidades Europeas, a la hora de suscribir el TUE, de un camino que abriera de forma más directa la «Unión Europea», donde se reconoce vinculado a la ciudadanía de los Estados miembros la ciudadanía de la Unión; y quizás no menos importante, los pasos que se dan en políticas comunitarias que hasta la fecha eran no sólo de los Estados, sino referentes del Estado como organización política (Política Exterior y de Seguridad Común, Políticas de Cooperación Policial y Judicial en materia Penal, moneda única, etc) hacen más que oportuno abrir un mayor nivel de intervención de Parlamento en el funcionamiento institucional de la Unión. A pesar de ello, como veremos, claramente insuficiente. La Declaración número 13 relativa al cometido de los Parlamentos nacionales se manifiesta partidaria de intensificar la información entre los parlamentos nacionales y el Parlamento europeo, por dos vías:

Intensificando el intercambio de información entre los Parlamentos nacionales y el Parlamento Europeo. En ese contexto, los Gobiernos de los Estados miembros velarán, entre otros aspectos, por que los Parlamentos nacionales puedan disponer de las propuestas legislativas de la Comisión con la antelación suficiente para su información o para que puedan ser examinadas.

La Conferencia considera asimismo importante que se intensifiquen los contactos entre los Parlamentos nacionales y el Parlamento Europeo, en particular gracias a la concesión de facilidades mutuas adecuadas y mediante reuniones periódicas de parlamentarios interesados por las mismas cuestiones.

Esta es una modestísima aproximación a la participación de los parlamentos nacionales en la acción política de la Unión Europea. De muy escasa repercusión, al menos por dos motivos: primero, deja en manos de los Gobiernos de los Estados que dispongan cómo informan de los asuntos Europeos a sus parlamentos; y segundo, se habla de que se informará de las propuestas legislativas de la Comisión pero no se determina a qué tipo de propuestas se refiere, y si debe ser en un tiempo determinado y si ese conocimiento puede llevar consigo algún tipo de manifestación de la Cámara.

La Declaración Número 13 se completa con otra sobre las Conferencias de los Parlamentos donde parece decantarse por la creación de un órgano específico para organi- 
Comunitaria. No olvidemos que se constituye con un número muy reducido de diputados de cada cámara nacional — tres_-, que desarrolla su trabajo durante un periodo de tiempo muy breve — día y medio—y que no se reúne más que dos veces al año ${ }^{11}$.

El Tratado de Ámsterdam también refuerza la presencia de los parlamentos nacionales en la política comunitaria ${ }^{12}$. Frente a la declaración de Maastricht, que como veíamos más arriba, tan sólo hacía referencia a la necesidad de fomentar la participación de los parlamento nacionales y que los gobiernos velaran porque aquellos tuvieran con suficiente antelación las propuestas legislativas de la Comisión para su conocimiento y examen, el Protocolo número 9 convierte la recomendación en una obligación para la Comunidad y los Gobiernos y, además, en una obligación con un plazo determinado:

«I. Información a los Parlamentos nacionales de los Estados miembros.

1. Todos los documentos de consulta de la Comisión (libros blancos y verdes y comunicaciones) se transmitirán puntualmente a los Parlamentos nacionales de los Estados miembros.

2. Las propuestas legislativas de la Comisión, definidas como tales por el Consejo de conformidad con el apartado 3 del artículo 151 (en la actualidad 207) del Tratado constitutivo de la Comunidad Europea, se comunicarán con la suficiente antelación para que el Gobierno de cada Estado miembro pueda velar por que su Parlamento nacional las reciba convenientemente.

3. Entre el momento en que la Comisión transmita al Parlamento Europeo y al Consejo una propuesta legislativa o una propuesta de una medida que deba adoptarse en virtud del Titulo VI del Tratado de la Unión Europea en todas sus versiones lingüisticas y la fecha de inclusión de dicha propuesta en el orden del día del Consejo para que éste decida al respecto bien la adopción de una acto, o bien la adopción de una posición común de conformidad con los artículos 189 B o 189 C (los actuales arts. 251 y 252) del Tratado constitutivo de la Comunidad Europea, deberá transcurrir un plazo de seis semanas, salvo excepciones por motivos de urgencia, debiendo mencionarse éstos en el acto o la posición común.»

Como decía, es un Protocolo que convierte las recomendaciones de informar que preveía Maastricht en un deber vinculante para la Comisión y los gobiernos de los Estados. No menos importante es que se abra un tiempo entre la tramitación por parte de la Comisión de una propuesta legislativa al Parlamento y el Consejo de seis semanas que, en definitiva, se convierte en tiempo a favor de los parlamentos nacionales para que puedan conocer de las propuestas y mostrar su parecer ante sus gobiernos que luego expresarán su punto de vista en el Consejo.

La preocupación por la posición en la arquitectura institucional Europea de los parlamentos nacionales se mantiene, aunque no se haga modificación alguna, en el Tratado de Niza. En concreto, la Declaración 23, relativa al futuro de la Unión, recogida en el Acta Final, señala que tras un informe que se presentará en Gotemburgo en junio de 2001, el Consejo Europeo aprobará, en su reunión de Laeken/Bruselas en diciembre de 2001, una declaración que incluya iniciativas, entre las que se recoge: «la función de los Parlamentos nacionales en la arquitectura europea».

La Declaración de Laeken expresa la necesidad de abrir una Conferencia Intergubernamental para 2004 que, como todos saben, se adelantó al otoño de 2003 (se inicia

9 Puede consultarse el Working Document del Parlamento Europeo «Perspectives for cooperaticon between de European Parliament and the national parliaments. W-19, 1996. 
seguir reuniéndose, sino que en el Tratado de Ámsterdam consigue que se le reconozca expresamente en los apartados 4 a 7 del Protocolo número 9. En concreto, el Apartado II recoge la Conferencia de Órganos Especializados en Asuntos Europeos en los siguientes términos:

4. La Conferencia de Órganos Especializados en Asuntos Europeos, en lo sucesivo denominada COS AC, creada en París los días 16 y 17 de noviembre de 1989, podrá dirigir a las instituciones de la Unión Europea cualquier contribución que juague conveniente, basándose, en particular, en proyectos de textos normativos que los representantes de los Gobiernos de los Estados miembros decidan de común acuerdo transmitirle, en función de la naturaleza de los asuntos que traten.

5. La COS AC podrá estudiar cualquier propuesta o iniciativa legislativa relacionada con la creación de un espacio de libertad, seguridad y justicia que pueda tener consecuencias directas en los derechos y libertades de las personas. Se informará al Parlamento Europeo, al Consejo y a la Comisión de cualquier contribución realizada por la COSAC con arreglo al presente punto.

6. La COSAC podrá dirigir al Parlamento Europeo, el Consejo y la Comisión cualquier contribución que juague conveniente sobre las actividades legislativas de la Unión, en particular respecto de la aplicación del principio de subsidiariedad, el espacio de libertad, seguridad y justicia, asi como respecto de las cuestiones relativas a los derechos fundamentales.

7. Las aportaciones de la COSAC no vincularán en absoluto a los Parlamentos nacionales ni prejuzgarán su posición.

El reconocimiento de la COSAC mediante un Protocolo anexo, que como todos sabemos, tiene valor normativo en el Derecho Comunitario, le da a la Conferencia una gran relevancia, frente a la que tenía en Maastricht donde era reconocida mediante una Decisión que tan sólo tiene valor interpretativo. Sin embargo, ello no nos puede llevar a pensar que la COSAC haya pasado a formar parte de los órganos o instituciones de la Unión Europea. Más bien, como ha señalado Delgado-Iribarren, no dejamos de estar ante una «conferencia» interparlamentaria de cooperación, muy alejada de los «comités» existentes en la Unión $^{10}$. Una Conferencia con una fuerte consolidación organizativa y una importante función consultiva, pero que, sin embargo, no deja de tener una escasa repercusión desde el punto de vista de la participación de los parlamentos nacionales en la política

10 Delgado-Iribarren señala que la COSAC a pesar de su apariencia no deja de ser lo que su propia denominación indica, esto es, una «conferencia» interparlamentaria de cooperación, muy alejada de los «comités» consultivos existentes (económico y social, de las regiones...). Más bien se trata de la institucionalización de una conferencia internacional. DELGADO-IRIBARREN García-Campero, Manuel. La funcion de los parlamentos... Opus cit. Pág. 379.

11 Borrel, Carnero y López Garrido nos recuerdan que este órgano, de carácter estrictamente deliberativo, puede presentar propuestas a los presidentes de la instituciones comunitarias. Pero, aun siendo tomadas por unanimidad, sus contribuciones son tan poco relevantes que ni siquiera se consideran que vinculen a los Parlamentos cuyos representantes las aprueban. BORREL, José; Carnero, Carlos y López Garrido, Diego. Construyendo la Constitución Europea. Crónica Política de la Convención. Edit. Real Instituto Elcano. Madrid 2003, pág. 138.

12 Aunque para parte de la doctrina esta participación sigua siendo insuficiente. En esa línea Mangas Martín señala que no se han abordado alternativas serias a la participación de los Parlamentos nacionales ni se ha estimulado la participación de los Parlamentos nacionales. Los ámbitos en los que los Tratados llaman a la participación de los Parlamentos nacionales siguen siendo una falacia y cuando es real, es insuficiente. MANGAS MARTíN, ARACELI. «La reforma institucional...» opus cit., pag. 23. 
los parlamentos nacionales:

- ¿Deben estar representados en una nueva institución, junto al Consejo y el Parlamento Europeo?

- ¿Deben desempeñar una función en los ámbitos de acción europea en los que no es competente el Parlamento Europeo?

- ¿Deben centrarse en el reparto de competencias entre la Unión y los Estados miembros, por ejemplo, mediante el control previo respecto del principio de subsidiariedad?

Las tres preguntas introducen en la Convención un tema que ya había sido planteado por parte de la doctrina y, en especial, por algunos parlamentos nacionales, ¿no sería conveniente crear en el entramado institucional de la Unión una segunda cámara, que conformada por miembros de los parlamentos nacionales hiciera presente a éstos y contribuyese a la «parlamentarización» de las decisiones de la Comunidad? ${ }^{16}$.

Los partidarios de esta solución dicen que de este modo se podría ver reforzada la legitimidad democrática de la Unión y se podría responder a la preocupación de que los parlamentos nacionales vean cada día más reducida su influencia en la toma de decisiones europeas. El Informe de Giorgio Napolitano para la Convención no se muestra partidario de esta solución con argumentos del siguiente tenor: estaríamos ante una nueva institución no elegida directamente por los ciudadanos (como ya lo es el Consejo). No sabemos cuales serían sus poderes, consultivos, de decisión o de control. En el supuesto de que fueran poderes de control, acabarían anulando los de los parlamentos nacionales frente a sus respectivos Gobiernos; si se tratan de poderes de Codecisión legislativa, se podrían sobreponer en un sistema complicado a los atribuidos al Parlamento europeo. Todo ello, sin entrar a barajar si el Consejo estaría dispuesto a que aquella actividad que no está fiscalizada por el Parlamento europeo pudiera ser fiscalizada por ésta segunda cámara. En definitiva, el Informe concluye señalando que la opción solucionaría pocos problemas y sin embargo, si que volvería más pesada, si cabe, la arquitectura institucional de la Unión.

Otra hipótesis fue la que propuso el Primer Ministro francés Jospin consistente en crear una «Conferencia permanente de los Parlamentos o Congreso», que se reuniera en sesiones periódicas para comprobar si se respeta el principio de subsidiariedad y para debatir cada año sobre el «estado de la Unión», además de para ejercer poderes de ratificación de determinadas modificaciones de los Tratados. Ésta propuesta fue tenida en cuenta, tanto en el Informe de Giorgio Napolitano, como en las Conclusiones del Grupo IV de la Convención. Hasta tal punto que en el apartado V, punto 33, se muestran partidarios de que la Convención examine el modo en el que los parlamento nacionales podrían participar más en la configuración de la agenda y la estrategia políticas de la UE. El Grupo consideró que un mecanismo de debate europeo en el que participaran tanto los parlamentos nacionales como el Parlamento europeo podría ser un formato adecuado. Además, se declara, para rechazar la opción de una segunda cámara, que ese nuevo foro no debería constituir una nueva institución, sino que sería un mecanismo de debate. No tendría funciones legislativas ni competencia alguna respecto de la delimitación de competencias entre la Unión y los Estados miembros ni perturbar el actual equilibrio institucional.

La posición de los miembros del Grupo IV se manifestó dividida ante el impulso de 
el 4 de octubre), y en donde entre los cuatro grandes temas a discusión, está el papel de los parlamento nacionales. Sin embargo, no se dan razones de peso más allá de argumentos genéricos como la necesidad de una mayor legitimidad democrática en las instituciones europeas. En concreto, se formulan preguntas del siguiente tenor: ¿Deben estar representados en una nueva institución, junto al Consejo y el Parlamento Europeo? ¿Deben desempeñar una función en los ámbitos de acción europea en los que no es competente el Parlamento Europeo? ¿Deben centrarse en el reparto de competencias entre la Unión y los Estados miembros, por ejemplo, mediante un control previo del respeto del principio de subsidiariedad? Sin duda, que el impulso de intervención de los parlamentos nacionales en la política europea, que viene desde el Tratado de Maastricht, tendrá alguna influencia en estas preguntas y las soluciones que se pretenden buscar; sin embargo, no son más que planteamientos genéricos y, como ha señalado la doctrina ${ }^{13}$ las razones de peso las debemos buscar en fuentes indirectas. En concreto, el documento elaborado por la Comisión de Asuntos Constitucionales del Parlamento Europeo. Donde se hace una clara referencia a las exigencias políticas de los Länder y de las demás regiones dotadas de poderes legislativos. Aunque el tan traído y llevado déficit democrático es importante y ha hecho avanzar en el fortalecimiento de los parlamentos, ahora aparece otra razón con no menos sustento ideológico y seguro que con más carga de interés político que hace mover la relevancia de los Parlamentos: la participación de los parlamentos regionales en los Estados federales o autonómicos. El argumento para los Estados Federales como Alemania es fácil y contundente: los Länder tienen competencias legislativas exclusivas en determinadas materias y de no participar en la formación de la voluntad de los representantes del Gobierno federal en el Consejo de la Unión se da la paradoja de que las competencias legislativas de los Länder serían «gestionadas» por las autoridades federales ${ }^{14}$. La solución, como es sabido es que los Länder tengan voz a través del Bundesrat, al formar la voluntad del Gobierno federal, y a las reuniones de los Consejos de la Unión acudan representantes del Gobierno federal y de los Länder, evidentemente, ambos son representantes del Gobierno federal y hablan con una sola voz a los efectos de la Unión.

La importancia de cuestiones como la que acabamos de señalar hacen que en todos los trabajos preparatorios esté clara la idea de que uno de los temas a tratar en la Convención de 2004 tenía que ser el papel del Parlamento europeo y los parlamentos nacionales. Giorgio Napolitano, Presidente de la Comisión de Asuntos Constitucionales del Parlamento europeo presenta un documento de trabajo sobre las relaciones entre el Parlamento europeo y los parlamentos nacionales en la construcción europea, donde se tratan cuestiones como el «déficit democrático y el papel de los Parlamentos», los problemas de la «parlamentarización» en el desarrollo de la Unión Europea o, entre otros, las relaciones entre los parlamentos nacionales y Gobiernos nacionales ${ }^{15}$. Cuestiones éstas, que junto con los interrogantes que se hacía la Declaración 23 del Tratado de Niza ayudan a conformar las preguntas que según Laeken debía responder la Convención a propósito de

13 Vid el trabajo de LINDE PANIAGUA, ENRIQUE. «Los parlamentos nacionales y regionales y su implicación en la toma de decisiones de la Unión Europea». En la Revista de Derecho de la Unión Europea, núm. 6. Primer semestre 2004. Págs 163-164.

14 Ibidem, pág 163.

15 En esta cuestión se ha suscitado si no se estaría estableciendo una nueva vía para restar protagonismo al Parlamento Europeo. Matia Portilla ha señalado como en dicho proceso la posición del Parlamento Europeo ha sido de reclamar competencias de control al mismo tiempo que se traspasan competencias a los Estados. 
control en cada país dependerá de las pautas constitucionales y forma de concebir la relaciones gobierno parlamento en cada Estado, y por ello el Informe no establece pautas comunes. Por este motivo el Informe no entiende conveniente entrar en un estudio comparado de instrumentos de control, pero si que introduce ciertas recomendaciones que podrían favorecer el control:

- La puntualidad, el alcance y la calidad de la información referente a todas las actividades de la Unión.

- La posibilidad de que un parlamento nacional formule su posición respecto de una propuesta de medida o acto legislativo de la Unión Europea.

- Los contactos y comparecencia periódicas de ministros antes y después de las sesiones del Consejo, así como de la sesiones del Consejo Europeo.

- La participación activa de comisiones permanentes o sectoriales en el proceso de control.

- Los contactos periódicos entre parlamentarios nacionales y diputados al Parlamento Europeo.

- La disponibilidad de personal de apoyo, incluida la posibilidad de contar con una oficina de representación en Bruselas.

El Informe del Grupo observa que quizás a quién le correspondería elaborar recomendaciones para ese mejor control por parte de los parlamentos es a la COSAC, y para ello, insta a la Presidencia Danesa a que en la propuesta para la reforma de la COSAC introduzca esta cuestión. Por otro lado, se le requiere que elabore un código de conducta o directrices de los parlamentos nacionales para ejercer el control parlamentario del trabajo de la Unión. En esta línea la XXVIII conferencia de la COSAC celebrada en Bruselas el 27 de enero de 2003 aprueba las Orientaciones de Copenhague para las relaciones entre los gobiernos y los parlamentos en los asuntos comunitarios y europeos ${ }^{17}$ :

Otra cuestión importante reflejada en el Informe es el acceso a los asuntos de la Unión por parte de los parlamentos nacionales. Hasta ahora la remisión de información a los parlamentos correspondía a los gobiernos de cada Estado. En el Informe se parte del principio de que deben ser éstos los que tengan encomendada de forma principal esta misión, sin embargo, ello no debe impedir que esos documentos y propuestas legislativas puedan ser enviadas directamente desde la Comisión ${ }^{18}$. Igual recomendación se hace respecto de los Libros Verdes y Libros Blancos y Comunicaciones ${ }^{19}$.

El Protocolo Anexo al Tratado de Ámsterdam sobre el cometido de los parlamentos

Dicho autor, sostiene que dicha posición olvida que la principal fuente de legitimación de la Unión hoy por hoy siguen siendo los Estados y, por tanto, no debe de extrañar que los parlamentos nacionales quieran incidir en los asuntos europeos. Matia Portilla, Francisco Javier. «Parlamento Europeo y parlamentos nacionales: doble legitimidad en el marco de la Unión Europea». En la REP, núm. 119, pág.200. Véase el documento de Giorgio Napolitano «Documento de trabajo sobre las relaciones entre Parlamento Europeo y Parlamentos nacionales en la construcción europea», puede consultarse adjunto al trabajo de Enrique Barón «El debate sobre el futuro de Europa», en la obra de AA. VV. La encrucijada constitucional de la Unión Europea, opus cit. Págs. 427-435.

16 Vid. Joel RideAu. «El papel de los parlamentos nacionales en la arquitectura europea». En la obra de AA.VV. La encrucijada constitucional de la Unión Europea, opus cit. Págs. 327-350 
este nuevo foro que denominaban sus partidarios «Congreso», y sus detractores no veían en él más que la voluntad de aquellos partidarios de la segunda cámara pero con un nivel rebajado puesto que no había podido imponerse como institución y, lo que era más importante, un foro que vendría a competir con la COSAC. Por lo tanto, aunque no se descartó la constitución de dicho foro si que se deja constancia de que debe estudiarse con detenimiento la relación con la COSAC. Posición que, como veremos, es la que va a mantener el Protocolo sobre el cometido de los Parlamentos nacionales en la Unión Europea.

\section{INFORME FINAL DEL GRUPO IV SOBRE EL COMETIDO DE LOS PARLAMENTOS NACIONALES EN LA UNIÓN EUROPEA}

Las conclusiones del Grupo IV parten de una idea consolidada en la doctrina y en los documentos de la Unión Europea desde hacía años: es necesario reforzar la participación de los parlamentos nacionales en el trabajo de la Unión. Para conseguir dicho objetivo el Informe se organiza sobre tres grandes cuestiones que, en parte, tienen que ver con las tres preguntas que se hacía la Declaración de Laeken:

- Papel de los parlamentos nacionales en el control de los gobiernos (sistema de control nacional)

- Papel de los parlamentos nacionales en la supervisión de la aplicación del principio de subsidiariedad

- Papel y función de las redes o mecanismos multilaterales de participación de los parlamentos nacionales a nivel europeo

Para poder afrontar estas tres preguntas el Informe parte de dos precisiones previas y claras. Primera, que potenciar el papel de los parlamento nacionales no tiene que ir en detrimento de las competencias del Parlamento europeo. El Parlamento europeo y los parlamentos nacionales tienen cometidos distintos en la organización de la Unión, pero ambos contribuyen a acercar la Unión Europea a los ciudadanos y a reforzar la legitimidad democrática de la Unión. Segunda, la participación de los parlamentos nacionales en la Unión contribuirá a abrir y dar transparencia a los trabajos del Consejo. Para el Informe el Consejo debe legislar «a puertas abiertas», y cuando lo haga a puertas cerradas debe explicar los motivos.

Como se puede observar, incluso en las precisiones previas ya aparecen los dos grandes problemas que Laeken y el Informe de la Comisión Constitucional planteaban para la Conferencia en materia parlamentaria: por un lado, la compatibilidad del trabajo de Parlamento europeo y los parlamentos nacionales, si el desarrollo de éstos últimos no generaría susceptibilidades e inconvenientes en el trabajo del primero. Como después veremos, no faltan en la doctrina los que piensan que así es. Por otro, responder a la pregunta de si es posible que el Consejo admita que se controle aquella actividad que realiza, entre ellas la legislativa, y que no está sometida al control del Parlamento europeo. También aquí más adelante veremos como se ha resuelto la situación.

Para responder a la primera pregunta, el papel de los parlamentos nacionales en el control de los gobiernos, se parte de la necesidad de que sea cada parlamento nacional el que se encargue del control de su respectivo gobierno. Ahora bien, cómo se articula ese 
nacionales contiene una disposición (art. I.2.) que señala que Las propuestas legislativas de la Comisión, definidas como tales por el Consejo de conformidad con el apartado 3 del artículo 207 del Tratado constitutivo de la Comunidad Europea, se comunicarán con la suficiente antelación para que el Gobierno de cada Estado miembro pueda velar por que su parlamento nacional las reciba convenientemente». Este era un asunto que ya habíamos comentado al tratar el Protocolo y allí ya se declaraba que esta mención a propuestas legislativas de la Comisión era una expresión demasiado inconcreta, por lo que el Informe se manifiesta por una mayor claridad para abarcar todas las propuestas de actos legislativos. Además, expresa la necesidad de que esas propuestas legislativas se transmitan directamente de la Comisión a los parlamentos nacionales al mismo tiempo que se envían al Consejo.

Otro asunto discutido del Protocolo Anexo a Ámsterdam es el del plazo que debe transcurrir desde la tramitación por la Comisión de una propuesta legislativa al Parlamento europeo y al Consejo y su inclusión en el orden del día del Consejo para que éste decida. El Punto I. 3 del Protocolo establecía que debía transcurrir un plazo de seis semanas, salvo excepciones por motivos de urgencia. El Informe entiende que este período es suficiente. Sin embargo, se plantea que en algunas ocasiones puede darse el caso de que los grupos de trabajo del Consejo lleguen a acuerdos preliminares antes de que los parlamentos nacionales hubieran podido dar a conocer su opinión a su Gobierno. Para evitar esto, durante ese período de seis semanas no debería reconocerse ningún acuerdo preliminar en el Consejo, ni en los grupos o el Coreper.

Finalmente, en la línea hasta aquí planteada de agilización en el conocimiento del trabajo de la Unión por los parlamentos nacionales se expresa la utilidad de que la Comisión remita su estrategia política anual y su programa legislativo y de trabajo anual, además de que el Tribunal de Cuentas remita su informe anual simultáneamente al Parlamento europeo, al Consejo y la los parlamentos nacionales.

La segunda cuestión. La aplicación del principio de subsidiariedad era y, quizás sigua siendo, uno de los asuntos principales. Para el Informe cuatro son las cuestiones esenciales:

- ¿Tienen una función los parlamentos nacionales en el control de subsidiariedad?

- ¿Deberían actuar aisladamente o con otros órganos?

- ¿En qué fase o fases del proceso legislativo deberían participar los parlamentos nacionales?

- ¿Qué mecanismo sería el más apropiado?

Para el Grupo IV de Trabajo está claro que en la aplicación del principio de subsidiariedad la responsabilidad debe estar compartida en la Comisión, el Parlamento europeo, el Consejo y los parlamentos nacionales. En concreto, sobre éstos últimos su actividad es fundamental para asesorar, controlar y pedir cuentas a sus gobiernos en cuanto a las actuaciones que desarrollen en el Consejo, pero desde luego su actuación la desarrollan individualmente o de forma conjunta con los órganos europeos que estén interviniendo.

Sobre el momento procesal oportuno para actuar por parte de los parlamentos nacionales, se decantan por una intervención en dos etapas: al inicio, cuando se tiene conocimiento del proyecto legislativo y, una vez que se inicia el procedimiento, mediante un seguimiento para controlar si las posibles modificaciones que se introduzcan afectan a la aplicación del principio de subsidiariedad. En caso de que así sea deben dar cuenta de ello 
al órgano correspondiente.

La tercera y última cuestión que el Informe se cuestiona es sobre cómo crear y mantener redes o contactos multilaterales de participación de los parlamentos nacionales.

No hay grandes aportaciones. Se parte de reconocer la importancia de estos contactos multilaterales. Entre otras razones, aunque no lo dice expresamente el Informe, porque de esta forma se avanza en una unificación de prácticas en procedimientos de conocimiento, control y toma de posición de los parlamentos en cuestiones europeas. Prácticas que podrían ser de aplicación al ámbito subestatal. Mención importante puesto que como todos sabemos la intervención de los parlamentos regionales o de los estados federados es un

17 Orientaciones de Copenhaqgue. La COSAC ha decidido indicar una serie de principios orientativos (o normas minimas) que permitirán garantizar que todos los parlamentos nacionales tengan la oportunidad de participar $e$ influir en los asuntos europeos de forma activa.

Es posible señalar tres elementos en las relaciones entre los gobiernos y los parlamentos que permitirán a los

Parlamentos nacionales aumentar su influencia en la política comunitaria.

Estos tres elementos son la calidad y la cantidad de la información que reciben los parlamentos nacionales, la gestión en el tiempo del intercambio de información y, finalmente, las oportunidades de las que disponen los parlamentos nacionales para utilizar la información recibida con el fin de aumentar su influencia en los asuntos comunitarios.

Se recomiendan los siguientes principios básicos con arreglo a los anteriormente expuesto:

- el parlamento nacional deberá recibir información relevante sobre las iniciativas comunitarias, tanto de parte del gobierno como de las instituciones comunitarias, a su debido tiempo de forma que tenga la oportunidad de examinarla antes de adoptar cualquier tipo de decisión,

- el parlamento nacional tendrá una oportunidad real de utilizar la información recibida para aumentar su ámbito de influencia en la politica europea de su propio país y, por tanto, de las decisiones comunes adoptadas en la Comunidad,

— el parlamento nacional podrá llevar a cabo el seguimiento de las decisiones de su propio gobierno en el marco del sistema comunitario.

Recomendaciones sobre las orientaciones generales (»Orientaciones de Copenhague»)

Se recomiendan las siguientes orientaciones generales con arreglo a los principios anteriormente expuestos:

1. El gobierno de un Estado miembro deberá garantizar, previa consulta con las instituciones comunitarias, que el parlamento nacional recibe toda la documentación relativa a la legislación e iniciativas de la Comunidad tan pronto como esté disponible.

2. El gobierno debería preparar material formulado de forma clara y que sea fácilmente accesible sobre cuestiones como la legislación comunitaria y ponerlo a disposición de los parlamentos nacionales.

Ejemplos:

— El gobierno puede remitir regularmente al parlamento nacional listas con los actos comunitarios recientes, documentos de las audiencias, mensajes, etc.

- El gobierno puede elaborar notas explicativas sobre todas las cuestiones comunitarias relevantes en un plazo determinado.

3. Debería propiciarse la celebración de reuniones a nivel ministerial en los parlamentos nacionales con suficiente antelación antes de las reuniones a escala comunitaria. En estas reuniones, el gobierno deberá informar sobre su posición con respecto a las propuestas comunitarias.

Ejemplos:

- El parlamento deberá tener la posibilidad de plantear preguntas a los ministerios con suficiente antelación antes de la celebración de las reuniones del Consejo a fin de conocer la posición del gobierno con respecto a determinadas cuestiones.

- La Comisión parlamentaria de Asuntos Europeos y una comisión de expertos deberían poder celebrar reuniones frecuentemente con la participación de un ministro y adaptadas a las reuniones del Consejo de forma que el parlamento pueda examinar con mayor concreción el contenido de dicha reuniones.

4. El gobierno debería informar al parlamento con suficiente antelación de las decisiones que deben adoptarse a escala comunitaria $y$ de las propuestas del gobierno en relación con dichas decisiones. Esto afecta a las reuniones ordinarias del Consejo, a las Cumbres y a las Conferencias Intergubernamentales. Los parlamentos nacionales deberían recibir información adecuada sobre las decisiones adoptadas.

Ejemplos:

- El gobierno puede remitir los órdenes del día de las próximas reuniones del Consejo con las referencias pertinentes a los documentos legislativos de la Comunidad.

- El gobierno debe presentar las actas de las reuniones del Consejo con la mayor brevedad posible tras la conclusión de dichas reuniones. 
de Informes inferior a $1 / 3$ se limitará a motivar con más detalle la iniciativa respecto a la subsidiariedad. En el caso de recibir Informes de más de 1/3 de parlamentos, la Comisión debe revisar su propuesta. Después, la Comisión podrá optar por mantener o retirar la modificación.

Al menos dos temas no quedan claros en esta fase de conclusión de los Informes de los parlamentos nacionales. Primero, que parece que se quiere abrir la posibilidad a que no sea sólo el criterio numérico (Informes de 1/3 de parlamentos), sino también criterio de relevancia de los argumentos de los informes que se presenten, los que determinen la opción que debe tomar la Comisión. Segundo, que cuando por el número o la importancia de los argumentos se insta a estudiar el impacto en la subsidiariedad de la iniciativa legislativa de la Comisión, ésta tendrá que revisar su propuesta pero no tiene que mantenerla, podrá retirar la iniciativa legislativa.

- Cuarto. Se propone no sólo que los parlamentos nacionales conozcan en el momento de la iniciativa legislativa, sino que también cuando tiene que intervenir el Comité de Conciliación para concordar las posturas de la Comisión con las del Consejo y las enmiendas del Parlamento europeo, los parlamentos nacionales pueden mostrar su opinión y parecer.

- Quinto. Para reforzar la intervención de los parlamentos nacionales en la garantía de la subsidiariedad se recomienda en el Informe que en el caso de que se haya puesto en marcha el procedimiento de «alerta rápida» y persista la discrepancia sobre la competencia se debería dar legitimación a dichos parlamentos para que pudieran interponer recurso ante el Tribunal de Justicia. Se propone legitimar a un parlamento nacional (o a ambas cámaras en caso de ser bicameral). También se propone se de competencia al Comité de las Regiones, evidentemente sobre aquellas materias de las que debe emitir dictamen.

La conclusión es que el Grupos I «sobre la subsidiariedad» se muestra favorable a que los parlamentos nacionales intervengan en todo el proceso legislativo de la Unión para garantizar que se cumplen los principios de subsidiariedad y proporcionalidad. Intervención

5. En cuanto a la asistencia administrativa en los parlamentos nacionales, compete a cada uno de ellos garantizar una óptima utilización de estas orientaciones, inter alia, fortaleciendo la asistencia administrativa y de expertos prestada al parlamento en asuntos europeos y adaptando esta asistencia a sus necesidades reales.

18 Apartado 13 del Informe.

19 Apartado 14 del Informe. 
problema de primera magnitud.

El intercambio de buenas prácticas e información entre parlamentos tiene su mejor ubicación en el trabajo que desarrolla la COSAC. Este organismo es el ámbito más adecuado para los debates entre parlamentarios nacionales y parlamentarios europeos. Se recuerda que ya el Protocolo anexo a Ámsterdam reconocía a la COSAC que pudiera dirigir a las instituciones de la Unión Europea «contribuciones» que juzgase convenientes (apartado II.4). El Informe recomienda que esta posibilidad sea completada con la necesidad de que las instituciones tuvieran que responder a la «contribuciones y observaciones».

Más allá de lo hasta aquí dicho, no se hacen más que propuestas genéricas sobre la constitución de foros de debate para orientar la política general y la estrategia de la Unión, así como conferencias interparlamentarias sobre cuestiones específicas.

\section{INFORME FINAL DEL GRUPO I SOBRE LA APLICACIÓN DE LOS PRINCIPIOS DE SUBSIDIARIEDAD Y PROPORCIONALIDAD}

En estrecha relación con lo que establece el Grupo de los parlamentos nacionales están las recomendaciones vertidas por el Grupo I «Subsidiariedad» (CONV 286/02). La primera aportación que hace el Grupo es reconocer que la aplicación del principio de subsidiariedad incumbe a todas las instituciones que participan del proceso legislativo, cosa que, como veíamos, también se señaló por parte del Grupo IV «Parlamentos nacionales». Pero lo que más nos interesa a nosotros en este momento es que la segunda aportación. La necesidad de instaurar un mecanismo de alerta rápida (early warning system) de naturaleza política encaminado a fortalecer el control del respeto del principio de subsidiariedad por parte de los parlamentos nacionales.

El Informe del Grupo I establece un procedimiento para que los parlamentos nacionales puedan velar por la correcta aplicación del principio de subsidiariedad por parte de todas las instituciones que participan en el procedimiento legislativo. Dicho procedimiento se organiza de la siguiente forma:

- Primero. La Comisión envía a cada parlamento nacional (allí donde haya dos cámaras a ambas) y al mismo tiempo que al Consejo y el Parlamento europeo la propuesta legislativa que elabora.

- Segundo. Desde la fecha de la trasmisión de propuesta legislativa los parlamentos nacionales tienen un plazo de seis semanas, en el que no puede iniciarse el procedimiento legislativo ni el Consejo podrá tomar acuerdos preliminares, para emitir un dictamen motivado sobre el principio de subsidiariedad. Dicho dictamen se envía a los presidentes del Parlamento europeo, Consejo y Comisión. Su contenido hará referencia tan sólo a aquellas cuestiones que afecten a la subsidiariedad y podrá ser de carácter general o referido a una disposición específica. También puede referirse a posibles modificaciones de las disposiciones que puedan afectar al principio de subsidiariedad.

- Tercero. Las consecuencias de dichos dictámenes en el proceso legislativo en una fórmula excesivamente genérica e indeterminada se dice que estarán en función de la cantidad y de su contenido. En el supuesto de recibir la Comisión un número 


\section{LA OPCIÓN DE LOS PROTOCOLOS DEL TRATADO CONSTITUCIONAL. LA NORMALIZACIÓN DEL PROCEDIMIENTO DE «ALERTA RÁPIDA».}

Tanto el Protocolo sobre el cometido de los parlamentos nacionales como el de la aplicación de los principios de subsidiariedad y proporcionalidad expresan, claramente, la línea de continuidad que en lo referido a la participación y control por parte de los parlamentos nacionales de la política comunitaria se ha desarrollado desde la ya lejana Declaración del Tratado de Maastricht.

Sin embargo, si que hay dos aportaciones que merecen ser tenidas en cuenta. Primera, que se ha optado de una forma decidida por constituir un procedimiento de «alerta rápida» para garantizar que los parlamentos nacionales pueden pronunciarse sobre si las iniciativas legislativas de la Comisión afectan al principio de subsidiariedad. Segunda, y no menos importante, que dicho procedimiento se reconoce en el articulado de la Constitución cuando en el Titulo III, de las Competencias de la Unión, en el Punto I.11, de los Principios Fundamentales, se establece en el apartado $3^{\circ}$ que los parlamentos nacionales velaran por el respeto a dicho principio (de subsidiariedad) con arreglo al procedimiento establecido en el mencionado Protocolo.

Como veíamos en el epígrafe anterior, relativo a las conclusiones del Grupo IV de la Convención, «...el respeto del principio de subsidiariedad y proporcionalidad es una responsabilidad compartida en la que Comisión, el Parlamento Europeo, el Consejo y los parlamentos nacionales deben velar por el cumplimiento del principio de subsidiariedad al proponer y examinar proyectos legislativos.» Pero no sólo se suman los parlamentos nacionales al conjunto de órganos que debe velar por el cumplimiento del principio de subsidiariedad, sino que más adelante se declara que «...los parlamentos nacionales han de desempeñar un papel esencial a la hora de contribuir a la labor de los órganos legislativos de la UE en la aplicación efectiva del principio de subsidiariedad.» En particular, asesorando, controlando y pidiendo cuentas a los ministros de su Gobierno respecto de su posición en el Consejo.

Con la entrada en vigor de la Constitución el principio de subsidiariedad que es fundamental para ordenar el reparto de competencias concurrentes entre la Comunidad Europea y los Estados miembros incorpora un procedimiento de aplicación y supervisión política para su cumplimiento donde los parlamentos nacionales se convierten en piezas esenciales $^{20}$. Pues bien, de acuerdo con el Protocolo, para que esta declaración sea efectiva se han de cumplir tres tipos de medidas:

-Que los parlamentos nacionales reciban las propuestas normativas de la Comisión directamente y al mismo tiempo que se envían al Parlamento europeo, al Consejo y a los Gobiernos de los Estados (apartados 1 y 2 del Punto I, Protocolo parlamentos nacionales).

- Que se garantice un período de tiempo suficiente para que los parlamentos nacionales puedan emitir su informe antes de la perfección del acto comunitario (apartado 4 del Punto I, Protocolo parlamentos nacionales).

- Que la Comisión tendrá que considerar nuevamente sus medidas si $1 / 3$ de las cámaras nacionales se oponen a la propuesta hecha (Punto 6 del Protocolo Subsidiariedad).

Las dos primeras medidas son indicación expresa del Informe final del Grupo IV 
que se desarrolla en todo el procedimiento no sólo al inicio con el control de la iniciativa y que puede condicionar de forma importante la actuación de la Comisión, sobre todo si se tiene en cuenta que en última instancia si persiste la discrepancia los parlamentos pueden ir a la solución jurisdiccional. 
mientos para la ejecución del Derecho comunitario. Precisamente, en esta línea de respeto a la autonomía en la organización institucional, el apartado quinto del Protocolo finaliza diciendo: ...incumbirá a cada parlamento nacional o a cada cámara de un parlamento nacional consultar, cuando proceda, a los parlamentos regionales que posean competencias legislativas ${ }^{24}$.

\section{CONCLUSIONES}

Llegado el punto de hacer las conclusiones creo que es bueno recuperar la idea central que exponía al inicio. En cincuenta años de conformación del proyecto europeo se han hecho grandes avances, creo que esto no lo discute nadie. Además, en los últimos diez años se ha hecho mucho camino para la parlamentarización de la Unión Europea. Como decía en aquellas páginas introductorias, Maastricht introduce al Parlamento europeo en la función legislativa, al incorporar la Codecisión; Ámsterdam, lo pone casi en pié de igualdad con el Consejo al extender la Codecisión a más áreas de regulación. También será Ámsterdam quien abra la puerta a la intervención de los parlamentos nacionales para el control de la acción normativa de la Unión Europea. Finalmente, los Informes de los Grupos de Trabajo de la Convención, que orientarán los Protocolos sobre la materia que se incorporan al Tratado Constitucional van a intensificar y fortalecer la intervención de los parlamentos nacionales en la acción Comunitaria.

Se ha dicho en más de una ocasión que el fortalecimiento de los parlamentos nacionales es una forma encubierta de restar protagonismo y relevancia institucional al Parlamento europeo ${ }^{25}$. No lo creo. Hoy por hoy la fuente de legitimación de las instituciones europeas está más en los Estados que en el Parlamento europeo. Aunque se han hecho grandes avances en la línea de legitimación de la Unión desde el Parlamento europeo, todavía estamos en un modelo de doble legitimación: la estatal, que se expresa en la intervención de los Estados en el Consejo, y la resultante de la representación parlamentaria de los ciudadanos de los pueblos de la Unión que conforme el Parlamento europeo. Por ello, como los Estados siguen siendo el centro del poder en la Unión Europea es conveniente que los parlamentos de éstos se pronuncien sobre la actuación de las Instituciones europeas y, lo que es más importante, sobre la posición que toman sus Ejecutivos en la conformación de esas políticas. Por ello, como ha señalado Rideau, la relación del Parlamento europeo con los parlamentos nacionales se debe inscribir en una perspectiva de división de tareas. La Asamblea europea representa la «democracia» en el nivel europeo con el ejercicio de las funciones legislativas y de control que tiene encomendadas, y los parlamentos nacionales desarrollan la función de control de sus respectivos Gobiernos en el ámbito interno ${ }^{26}$.

Menos adecuado me parece, por razones de economía funcional y racionalidad institucional, que hubiera prosperado la creación de una segunda cámara donde estuvieran representados los parlamentos nacionales. Además de que ésta si que podría haber generado un

20 Sobre dichos principios se han escrito a lo largo de estos últimos años innumerables trabajos, por todos vid. Rubio Llorente, FANCISCO. «El principio de subsidiariedad y el principio de proporcionalidad», en la obra de AA.VV. La Constitución Europea. Dirigida por Marcelino Oreja. Actas del Curso de Verano de 1993 de la Universidad Complutense. Madrid 1994, págs. 213 y ss. También, Boixareu Carrera, Ángel. «Principales cuestiones planteadas por el principio de subsidiariedad». En la obra de AA.VV. La Constitución Europea, opus cit. Pa'gs. 231 y ss. 
preparatorio de la Convención. Allí ya se señala que los parlamentos nacionales deben tener acceso a los documentos y propuestas normativas de la Comisión directamente y al mismo tiempo que se ponen en circulación para los organismos internos y los Gobiernos. En el punto segundo, referido al tiempo necesario para la emisión de su posición por parte de los parlamentos nacionales, también allí se decía que sería de seis semanas y, además, que el Consejo o el Coreper no debería reconocer acuerdos preliminares en ese periodo de seis semanas que pudieran condicionar la decisión de aquellos. Sin embargo, la medida tercera es una novedad, que en la práctica aporta poco puesto que constatado el desacuerdo emitirá un segundo acuerdo que revitaliza la medida adoptada por la Comisión.

Finalmente, no se ha de olvidar, que el Protocolo reconoce la opción como último recurso de los parlamentos y sus cámaras para abrir la vía del control jurisdiccional mediante el recurso ante el Tribunal de Justicia. Posibilidad, que sin embargo, se reconoce en el Protocolo matizada respecto de la posición que sostuvo el Informe de Grupo I. Se dice que el Tribunal conoce de los recursos por incumplimiento del principio de subsidiariedad, de acuerdo con los procedimientos previstos en el artículo III-270 de la Constitución. Pero la legitimación no se otorga directamente a los parlamentos sino que se les reconoce a los Estados a instancia propia (del Ejecutivo) o a instancia del Parlamento, o de una cámara, de acuerdo con el procedimiento que en cada Estado se establezca. El Comité de las Regiones podrá interponer recurso sobre cuestiones donde la Constitución requiera su consulta ${ }^{21}$.

Es también reseñable cómo el Protocolo de la Subsidiariedad se ocupa de los efectos que las medidas legislativas que tome la Comisión puedan afectar a competencias de entes territoriales infraestatales en los Estados descentralizados (federales o autonómicos) ${ }^{22}$.

Aunque el punto dos señala que cuando la Comisión proponga un acto legislativo debe tener en cuenta la dimensión regional y local, esto no cambia nada respecto de los órganos a los que debe consultar: Consejo, Parlamento europeo y parlamentos nacionales. No debemos olvidar que está presente el principio de autonomía institucional por el que las formas de ordenar el control interno y la distribución de competencias internas no es una materia que deba determinar la Unión ${ }^{23}$. Incluso el Tribunal Constitucional español ya se pronunció sobre esta materia, para la aplicación del derecho derivado, cuando en la Sentencia 252/1988 señala que el Derecho comunitario no forma parte del bloque de la constitucionalidad y por ello el cumplimiento de las obligaciones derivadas de la pertenencia a la Unión Europea corresponde al ente interno, poder central o autonómico que tenga la competencia material. El Alto Tribunal español deja claro en su argumento que no se ha cedido a las Comunidades Europeas la competencia para modificar el reparto interno de poderes entre el Estado y las Comunidades Autónomas. Y además, el Derecho comunitario se organiza desde el respeto al principio de autonomía institucional que supone que es el Derecho de cada Estado el que debe determinar los órganos competentes y los procedi-

21 Vid. Díez-Hochleitner, Javier. «El sistema competencial de la Unión Europea en el Proyecto de Constitución elaborado por la Convención Europea». En la obra de AA.VV. El Proyecto de nueva Constitución Europea. Dirección de Enoch Albertí Rovira. Edit. Tirant lo Blanch Monografías 314, Valencia 2004. Págs. 248 y ss. y SÁNChez SÁez, Antonio José. «La «democratización» de las fuentes del Derecho en el Proyecto de Constitución Europea». En la obra de AA.VV. Una Constitución para la Ciudadanía Europea. coord. Gómez Corona y otros. Edit. Aranzadi. Navarra 2004. Págs. 277 y ss. 
cámaras, puestos que tenemos un modelo parlamentario imperfecto ${ }^{28}$; ¿Qué sucede cuando la decisión parlamentaria no se toma por cada una de las cámaras sino por una Comisión Mixta? En definitiva, este es un asunto pendiente que dará mucho más de qué hablar en los próximos años ${ }^{29}$. En particular, en países como el nuestro en el que su cámaras no cuentan más que con una Comisión Mixta para tratar los asuntos de la Unión y no existe procedimiento que de aplicación al mandato del Protocolo.

No menos importante será ver el grado de compromiso que los Ejecutivos adquieren con la posición que tomen los Parlamentos. Desde luego que políticamente quedará su posición fuertemente condicionada, pero desde el punto de vista jurídico, salvo que se exprese de forma directa como ya pasa en algunos países de la Unión, podríamos tener posiciones divergentes que nos llevarían a un conflicto institucional interno.

También se ha dicho del early warning system que además de retardar la función legislativa de la Unión es más que probable que los parlamentos nacionales lo aprovechen para hacer consideraciones que nada tengan que ver con la subsidiariedad ${ }^{30}$. Esto es cierto, pero sin embargo, no me parece que haya que verlo como un problema, en todo caso ya está la Comisión para desestimar los informes remitidos en esos términos, y si como una vía para seguir avanzando en el fortalecimiento democrático y de participación de los parlamentos en las cuestiones de la Unión Europea.

Todas estas, y quizás alguna más, son las grandes preguntas que hay que hacer al sistema de intervención de los parlamentos nacionales en los asuntos europeos. Por supuesto son las que hay que hacer al sistema de «alerta rápida» que inaugura el Protocolo sobre la aplicación de los principios de subsidiariedad y proporcionalidad, que frente a aquellos que lo ven como una forma de restar protagonismo al Parlamento Europeo en el sistema de la Unión me parece que es una manera acertada de hacer presente las dos legitimidades con las que se mueve hoy por hoy Europa. Mañana, ya veremos, estamos construyendo una organización política nueva y la mejor manera de hacerlo es dejando que sus instituciones tengan margen de acción para poder adaptarse a los cambios que se producen día a día.

22 Sobre la participación de los entes territoriales en la política Europea vid. AlBERTí RoviRA, ENOCH. Los parlamentos regionales en la Unión Europea. En la obra de AA.VV. La encrucijada constitucional de la Unión Europea. Págs. 351 y ss. de 1971.

23 Posición tradicional de Unión Europea desde la Sentencia International Fruti Company, de 15 de diciembre

24 Sobre el Derecho Europeo y el Estado autonómico, vid, por todos a DíEz-Picazo, Luis María. Constitucionalismo de la Unión Europea. Edit. Cuadernos Civitas, Madrid 2002. Pags. 190 y ss. PÉrez-Tremps, PABLO. «La ejecución del Derecho Comunitario por las Comunidades Autónomas». En la obra de AA.VV. Curso de Derecho Público de las Comunidades Autónomas. Directores. Jaime Rodríguez- Arana y Pablo García Mexía. Madrid 2003. págs. 255 y ss. Albertí RovirA, EnOCH. «Las regiones en el proyecto de la nueva Constitución Europea», opus cit. Págs . 449-483. 
grave conflicto de competencias con el Parlamento europeo. Por otro lado, no se debe olvidar que la intervención de los parlamentos nacionales es limitada. Conoce de iniciativas legislativas así como otros documentos de programación política o legislativa (libros blancos, verdes o comunicaciones) de la Comisión. En especial las iniciativas legislativas para poder, como hemos visto, comprobar que se aplica el principio de subsidiariedad.

El problema no es, desde mi punto de vista, de competencias, el problema está en cómo funciona ese nuevo sistema de control parlamentario. Es buena la idea de hacer partícipe a los parlamentos nacionales de las decisiones comunitarias, ahora la cuestión es determinar cómo se hace.

El procedimiento de «alerta rápida» supone un paso más en la parlamentarización de la Unión Europea. Como decía antes, no estoy de acuerdo con que es una forma de restar protagonismo al Parlamento europeo por «la puerta pequeña». Es simple y llanamente atribuirles la posibilidad de que se pronuncien sobre una cuestión que afecta de forma directa a los Estados y a los entes territoriales de cada uno de ello: que las normas que pretende aprobar la Unión aplica correctamente el principio de subsidiariedad y no invade competencias que no le son propias.

Por otro lado, no debemos de olvidar que el Protocolo tan sólo reconoce una función y diseña los elementos fundamentales del procedimiento, pero han de ser los parlamentos nacionales los que organicen su desarrollo. Precisamente en este ámbito interno es donde será importante ver cómo se resuelven algunos de los grandes problemas que han quedado pendientes. Por ejemplo, la participación de los parlamentos regionales que poseen competencias legislativas. Como ya se ha dicho en páginas anteriores y ha señalado la doctrina ${ }^{27}$, el procedimiento plantea grandes interrogantes en esta materia. No se debe olvidar que el art. I.5.1 reconoce el hecho regional en la Unión y el principio de autonomía institucional está, también como hemos señalado antes, en los fundamentos de la Unión. Es cierto que en la línea victoriosa de los Estados las cuestiones regionales son asuntos internos. G. Napolitano, en el Informe sobre el papel de los poderes regionales, expresará con toda claridad la postura triunfante: únicamente los Estados son miembros de la Unión y establecen libremente su organización interna, en particular, la manera de asociar a sus colectividades locales a la toma de decisiones». Pero como decía, si es la cuestión regional un asunto interno, como ha señalado Albertí Rovira, ¿qué hace la Unión estableciendo que es el parlamento estatal el que debe tomar la posición común? Y más, ¿qué hace la Unión decidiendo como cuenta la presencia de las cámaras en los parlamentos bicamerales? Por ejemplo, para España puede que se haya creado un problema con el sistema de reparto de votos en pié de igualdad para las dos

25 Linde Paniagua se cuestiona el motivo del incremento de funciones de los parlamentos nacionales y concluye que es una muestra más del viraje intergubernamental al que asistimos en la UE. Entiende que lo que se oculta con este reforzamiento de los parlamentos nacionales es eludir el reforzamiento del Parlamento europeo. De esta manera se está creando una ficción de que estamos a un proceso de legitimación democrática de la Unión. Linde Paniagua, ENRiQue. «Los parlamentos nacionales y regionales y su implicación en la toma de decisiones en la Unión Europea». Opus cit. Pág. 168.

26 Rideau avanza más diciendo que esta situación podría englobar un cierto nivel de cooperación entre los Parlamentos europeo y nacionales, teniendo estos últimos la misión de controlar a los Gobiernos en la elaboración de las posturas europeas y en la aplicación de las normas elaboradas por las instituciones de la Unión Europea. RIDEAU, JoEL. El Papel de los parlamentos nacionales... Opus cit. Pág. 335. 
27 Albertí Rovira muestra como en el debate constitucional de la Unión estuvieron presente las dos grandes posturas en cuanto al hecho territorial. Por un lado la posición regionalista que buscaba en la Convención (Conferencia de Presidentes, CALRE, Comité de las Regiones) un lugar en la Unión, que consistiría en el reconocimiento de un estatuto de región asociada a la Unión que se aplicaría a los territorios que designasen los Estados y que conllevaría ciertos derechos de participación en las políticas de la Unión (Informe Lamassoure). Por otro, la posición de los Gobiernos de los Estados, que sostenían que la cuestión regional es un asunto que debe dilucidarse en el espacio interno de los Estados (Informe G. Napolitano). Albertí Rovira, EnOCH. «Las regiones en el proyecto de nueva Constitución Europea». En la obra de AA.VV. El proyecto de nueva Constitución Europea, opus cit. Pa'g. 458 y ss.

28 Ibidem, pág. 468. 
29 En nuestro país parece más que necesario un replanteo de cómo se cumple con el mandato del Protocolo de la Subsidiariedad. Desde luego ahora que nos empeñamos por la reforma del Senado y la integración en dicha cámara de las cuestiones territoriales parece lógico que este asunto se resolviese con la intervención de las Comunidades Autónomas en ella. Una posibilidad sería que el procedimiento de la Subsidiariedad pasase a formar parte de las competencias de la Comisión General de las Comunidades Autónomas del Senado.

30 Díez-Hochleitner, Javier. «El sistema competencial de la Unión Europea...», opus cit. Pa'g. 254. 
